\title{
Power Law Like Correlation between Condensation Energy and Superconducting Transition Temperatures in Iron Pnictide/Chalcogenide Superconductors: Beyond the BCS Understanding
}

\author{
Jie Xing ${ }^{1}$, Sheng $\mathrm{Li}^{1}$, Bin Zeng ${ }^{2}$, Gang $\mathrm{Mu}^{2}$, Bing Shen ${ }^{2}$, J. \\ Schneeloch $^{3}$, R. D. Zhong ${ }^{3}$, T. S. Liu ${ }^{3,4}$, G. D. Gu ${ }^{3}$, and Hai-Hu Wen ${ }^{1 *}$ \\ ${ }^{1}$ Center for Superconducting Physics and Materials, \\ National Laboratory of Solid State Microstructures and Department of Physics, Nanjing University, Nanjing 210093, China \\ 2 National Laboratory for Superconductivity, Institute of Physics and National Laboratory for Condensed Matter Physics, \\ Chinese Academy of Sciences, Beijing 100190, China \\ 3 Condensed Matter Physics and Materials Science Department, \\ Brookhaven National Laboratory, Upton, NY 11973, USA and \\ 4 School of Chemical Engineering and Environment, \\ North University of China, Shanxi 030051, China
}

(Dated: June 29, 2018)

\begin{abstract}
Superconducting condensation energy $U_{0}^{\text {int }}$ has been determined by integrating the electronic entropy in various iron pnictide/chalcogenide superconducting systems. It is found that $U_{0}^{\text {int }} \propto T_{c}^{n}$ with $n=3$ to 4 , which is in sharp contrast to the simple BCS prediction $U_{0}^{B C S}=1 / 2 N_{F} \Delta_{s}^{2}$ with $N_{F}$ the quasiparticle density of states at the Fermi energy, $\Delta_{s}$ the superconducting gap. A similar correlation holds if we compute the condensation energy through $U_{0}^{\text {cal }}=3 \gamma_{n}^{\text {eff }} \Delta_{s}^{2} / 4 \pi^{2} k_{B}^{2}$ with $\gamma_{n}^{\text {eff }}$ the effective normal state electronic specific heat coefficient. This indicates a general relationship $\gamma_{n}^{e f f} \propto T_{c}^{m}$ with $m=1$ to 2 , which is not predicted by the BCS scheme. A picture based on quantum criticality is proposed to explain this phenomenon.
\end{abstract}

PACS numbers: 74.70.Dd, 74.55.+v, 74.40.Gh

Superconductivity is induced by quantum condensation of large number of paired electrons, namely the Cooper pairs. According to the Bardeen-CooperSchrieffer (BCS) theory, the pairing is supposed to be established between the two electrons with opposite momentum and spins by exchanging phonons. The formation of the electronic paired state will lower the total energy leading to the condensation of the Cooper pairs. The condensation energy, defined as the the difference of the Gibbs free energy of the system in the normal state and superconducting state, is given by $U_{0}^{B C S}=1 / 2 N_{F} \Delta_{s}^{2}$ with $N_{F}$ the quasi-particle density of states (DOS) at the Fermi energy of the normal state, $\Delta_{s}$ is the superconducting gap. Suppose a simple and natural relation, $\Delta_{s} \propto T_{c}$, we have $U_{0}^{B C S} \propto N_{F} T_{c}^{2}$. Normally $N_{F}$ is weakly related to the superconducting gap $\Delta_{s}$ through $N_{F}=1 / V \ln \left[\left(2 \hbar \omega_{D}\right) / \Delta_{s}\right]$ with $V$ the attractive potential between the two electrons when exchanging a phonon and $\omega_{D}$ the Debye frequency, thus one can roughly expect that $U_{0}^{B C S} \propto T_{c}^{2}$ in a conventional BCS superconductor.

Since the discovery of iron based superconductors, the pairing mechanism remains unresolved yet. One type of picture assumes the similar scenario of the BCS but using the antifferomagnetic spin fluctuations as the pairing glue ${ }^{1-4}$. This is called the weak coupling approach. Another more exotic picture, based on the strong coupling approach, assumes the local magnetic interaction as the pairing force which simultaneously causes the pairing of two electrons $s^{-\frac{8}{-8}}$. However, both pictures will intimately lead to an $s^{ \pm}$pairing gap as the natural one. Specific heat $(\mathrm{SH})$ measurements are very powerful, not only in detecting the gap symmetry ${ }^{9}=11$, but also in unraveling some deeper mysteries related to the superconducting mechanism. For example, it was found by Bud'ko, Ni and Canfield (BNC) 12 that, in the 122 systems, there is a simple scaling relation $\left.\Delta C\right|_{T_{c}} \propto T_{c}^{3}$ with $\left.\Delta C\right|_{T_{c}}$ the $\mathrm{SH}$ anomaly (jump) at $\mathrm{T}_{c}$. This simple relation was later proved and solidified by further measurements with the samples experienced different thermal treatments and annealing 13 , and extended to the 11 and 111 systems also $\underline{14,15}$. This $\left.\Delta C\right|_{T_{c}} \propto T_{c}^{3}$ relation was explained as due to the impurity scattering effect in a multiband superconductor with the $s^{ \pm}$pairing gap $\underline{16}$. However, this explanation may suffer a challenge when making a comparison between $\mathrm{Ba}_{1-x} \mathrm{~K}_{x} \mathrm{Fe}_{2} \mathrm{As}_{2}$ and $\mathrm{Ba}\left(\mathrm{Fe}_{1-x} \mathrm{Co}_{x}\right)_{2} \mathrm{As}_{2}$ : the former is much cleaner than the latter judged through the residual scattering rate $\frac{17}{7}$, but they follow a similar trend in the scaling relation $\left.\Delta C\right|_{T_{c}} \propto T_{c}^{3}$. Another more novel picture, concerned with the quantum critical point $(\mathrm{QCP})^{18}$, was proposed to understand this interesting relation. Since the condensation energy is directly related to how much energy that is saved when the system enters the superconducting state, thus it is highly desired to have a systematic assessment on the condensation energy. In this Letter we try to calculate the condensation energy from 10 pieces of our measured single crystals, and others from the published literatures. Surprisingly we discovered a simple power law like relation between the condensation energy and the superconducting transition temperatures.

Single crystals of $\mathrm{Ba}_{1-x} \mathrm{~K}_{x} \mathrm{Fe}_{2} \mathrm{As}_{2} \quad(\mathrm{x}=0.3,0.4)$, $\mathrm{Ba}\left(\mathrm{Fe}_{1-x} \mathrm{Co}_{x}\right)_{2} \mathrm{As}_{2}, \quad \mathrm{BaFe}_{1.9} \mathrm{Ni}_{0.1} \mathrm{As}_{2}$ were grown by the flux method ${ }^{9} 19$, the $\mathrm{FeSe}_{0.5} \mathrm{Te}_{0.5}$ by a unidirectional solidification method ${ }^{20}$. The $\mathrm{SH}$ data 

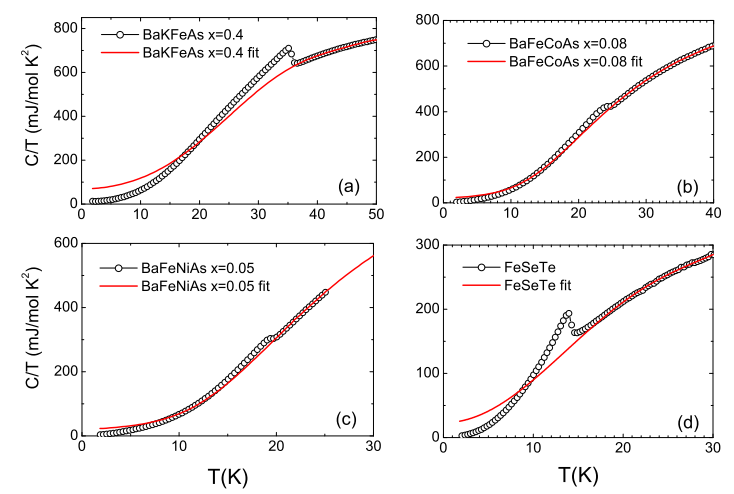

FIG. 1: (color online) Raw data of $\mathrm{SH}$ for four different superconducting systems near the optimal doping point. The data are shown for samples (a) $\mathrm{Ba}_{0.6} \mathrm{~K}_{0.4} \mathrm{Fe}_{2} \mathrm{As}_{2}$, (b) $\mathrm{Ba}\left(\mathrm{Fe}_{0.92} \mathrm{Co}_{0.08}\right)_{2} \mathrm{As}_{2} ;$ (c) $\mathrm{BaFe}_{1.9} \mathrm{Ni}_{0.1} \mathrm{As}_{2} ;$ and (d) $\mathrm{FeSe}_{0.5} \mathrm{Te}_{0.5}$. Here we show only four typical sets of data and the fitting curves of the normal state. More data are presented in the Supplementary Materials.

of $\mathrm{Ba}_{1-x} \mathrm{~K}_{x} \mathrm{Fe}_{2} \mathrm{As}_{2}(\mathrm{x}=0.4), \quad \mathrm{Ba}\left(\mathrm{Fe}_{1-x} \mathrm{Co}_{x}\right)_{2} \mathrm{As}_{2}$ and $\mathrm{BaFe}_{1.9} \mathrm{Ni}_{0.1} \mathrm{As}_{2}$ single crystals were published in previous papers $\frac{9.19}{}$. All doping concentrations of our samples are the nominal ones. The SH measurements were done by the thermal relaxation method on the physical property measurement system (PPMS, Quantum Design) with the advanced measuring chip. For determining the condensation energy, we properly removed the phonon contributions (see below). We also get the electronic SH data from the published papers of other groups $21-33$ so as to make the statistic results more convincing.

In Fig. 1, we present the temperature dependence of $\mathrm{SH}$ for four typical samples from the ten. The sharp $\mathrm{SH}$ anomaly can be seen clearly at $\mathrm{T}_{c}$ for each sample. In order to obtain the electronic $\mathrm{SH}$, we have to investigate the phonon part of the total $\mathrm{SH}$ carefully. For $\mathrm{Ba}\left(\mathrm{Fe}_{1-x} \mathrm{Co}_{x}\right)_{2} \mathrm{As}_{2}$ and $\mathrm{BaFe}_{1.9} \mathrm{Ni}_{0.1} \mathrm{As}_{2}$ samples, because the phonon contribution changes not much with doping, the overdoped nonsuperconducting samples are used as the references. Thus, from the formula

$$
C_{e}^{s}(T)=C_{\text {total }}^{s}(T)-p * C_{p h}^{n}(q * T)
$$

we can derive the electronic term in each superconducting sample. Here $\mathrm{C}_{e}^{s}(T), \mathrm{C}_{\text {total }}^{s}(\mathrm{~T})$ are the electronic and total $\mathrm{SH}$ of the superconducting samples respectively, $\mathrm{C}_{p h}^{n}(\mathrm{~T})$ is the phonon contribution of $\mathrm{SH}$ of the reference one. The $p$ and $q$ are fitting parameters which are determined by having a close matching effect of the phonon part between the superconducting sample and the reference one. It is found that $p$ and $q$ are close to $1, \underline{19}$ This slight modification of the phonon contribution is understandable since the doping may change the lattice constants slightly. For $\mathrm{Ba}_{1-x} \mathrm{~K}_{x} \mathrm{Fe}_{2} \mathrm{As}_{2}$ and $\mathrm{FeSe}_{0.5} \mathrm{Te}_{0.5}$ samples, we use a polynomial function $\mathrm{C}_{n o r}=\mathrm{C}_{e}+\mathrm{C}_{p h}=$ $\alpha \mathrm{T}+\beta \mathrm{T}^{3}+\gamma \mathrm{T}^{5}+\cdots$ to fit the data in the normal state

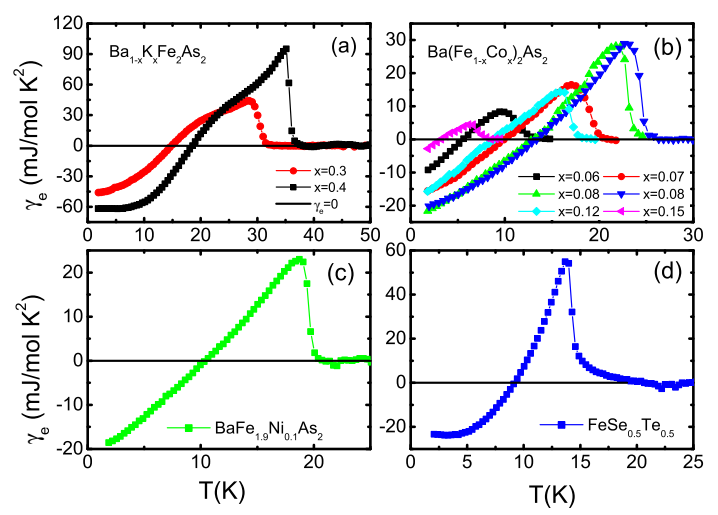

FIG. 2: (color online) Temperature dependence of the superconducting electronic specific heat shifted by $\gamma_{n}^{\text {eff }}+$ $\gamma_{0}$, i. e., $C_{e} / T-\gamma_{n}^{e f f}-\gamma_{0}$ for (a) $\mathrm{Ba}_{1-x} \mathrm{~K}_{x} \mathrm{Fe}_{2} \mathrm{As}_{2}$, (b) $\mathrm{Ba}\left(\mathrm{Fe}_{1-x} \mathrm{Co}_{x}\right)_{2} \mathrm{As}_{2} ;$ (c) $\mathrm{BaFe}_{1.9} \mathrm{Ni}_{0.1} \mathrm{As}_{2} ;$ and (d) $\mathrm{FeSe}_{0.5} \mathrm{Te}_{0.5}$.

above $T_{c}$. In the fitting process, to ensure the entropy conservation, we leave the electronic term $\alpha$ as the trying parameter and leave other higher-power temperature related terms totally free. The red lines in Fig. 1 show the phonon and the normal state electronic contribution of each sample. Either using a reference sample or using the polynomial fitting method, one can find a good fit of the normal state of each superconducting sample. We must emphasize that, to ensure the the entropy conservation is a basic rule we hold in removing the phonon contribution in either methods mentioned above. This may inevitably lead to some uncertainties of the condensation energy with the error bars of about $\pm 10 \%$. For clarity, we only show data for four optimally doped samples in Fig. 1 and the data of other six samples are presented in the Supplementary Materials (SM).

After subtracting the phonon contribution from the total $\mathrm{SH}$, the electronic contribution is obtained for our ten samples, as shown in Fig. 2. The residual term at $T=0 \mathrm{~K}$ gives actually the effective $\mathrm{SH}$ coefficient $\gamma_{n}^{e f f}=-\left(\gamma_{n}-\gamma(0)\right)$, with $\gamma_{n}$ the total electronic SH of the normal state, including the nonsuperconducting term $\gamma_{0} \stackrel{34}{\underline{3}}$ The $\mathrm{SH}$ anomaly at $\mathrm{T}_{c}$ rises to a maximum at optimal doping point with the highest $\mathrm{T}_{c}$. Above $\mathrm{T}_{c}$, the electronic SH decreases rapidly except for $\mathrm{FeSe}_{0.5} \mathrm{Te}_{0.5}$. For $\mathrm{FeSe}_{0.5} \mathrm{Te}_{0.5}$, there is a tail extending up to a higher temperature, which may suggest that this system can be made with higher transition temperatures, as achieved in thin films ${ }^{35}$. This phenomenon was found by other groups as well $21-24$.

According to the BCS theory, the $\mathrm{SH}$ anomaly of a superconductor at $\mathrm{T}_{c}$ should follow $\Delta \mathrm{C} / \gamma_{n} \mathrm{~T}_{c}=1.43$ in the weak coupling limit. However, it was found that the iron based superconductors severally violate this relation but show a simple correlation $\left.\Delta C\right|_{T_{c}} \propto T_{c}^{3}$. This power law seems to be appropriate for many iron based super- 


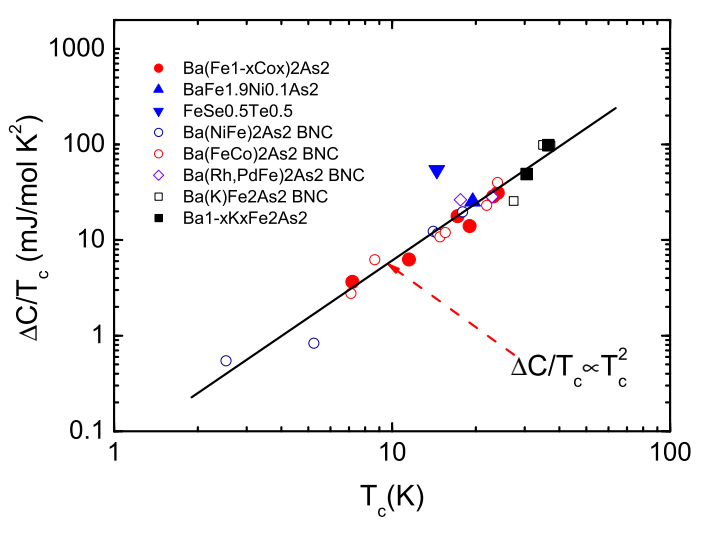

FIG. 3: (color online) Correlations between the SH anomaly at $T_{c}$, i.e., $\Delta C /\left.T_{c}\right|_{T_{c}}$ and $T_{c}$ for many iron based superconductors. The solid line shows the relationship with $\Delta C /\left.T_{c}\right|_{T_{c}} \propto$ $T_{c}^{2}$. The solid symbols are from our present experiment. The open ones are from the work of BNC.

conductors, with the majority of data so far for the 122 systems $12,14,15$. We also determined the SH anomaly of our ten samples and show them together with those of $\mathrm{BNC}$ in Fig. 3. Because of the finite width of the superconducting transition at $\mathrm{T}_{c}$, we use the entropy conservation to determine the value of $\mathrm{SH}$ anomaly and $\mathrm{T}_{c}$ in our samples. It's clear that our data fall onto the general power law $\left.\Delta C\right|_{T_{c}} \propto T_{c}^{3}$ quite well except for the $\mathrm{FeSe}_{0.5} \mathrm{Te}_{0.5}$ sample on which a deviation is observed. This may suggest that the general scaling law works better for one system, for example, for 122 here. However, we will show later that a scaling law of condensation energy with $\mathrm{T}_{c}$ seems more general to cover data from different systems, such as 122, 111 and 11 .

In addition to the $\mathrm{SH}$ anomaly, the condensation energy is another important parameter to determine the properties underlying the superconductivity. According to the thermodynamic definition, the entropy is $S=$ $-\partial G / \partial T$, therefore we can calculate the condensation energy by integrating the entropy of the superconducting and normal state,

$$
\begin{gathered}
U_{0}^{i n t}=\int_{0}^{T_{c}}\left(S_{n}(T)-S_{s}(T)\right) d T \\
=\int_{0}^{T_{c}} d T \int_{0}^{T}\left(C_{n}\left(T^{\prime}\right)-C_{s}\left(T^{\prime}\right)\right) / T^{\prime} d T^{\prime} .
\end{gathered}
$$

The temperature dependence of entropy is shown in the SM for the four optimally doped samples. Because $\left(C_{n}(T)-C_{s}(T)\right) / T=\gamma_{n}(T)-\gamma_{s}(T)$, we can just compute condensation energy with the electronic SH. We also calculate the condensation energy using the electronic $\mathrm{SH}$ data in previously published papers $21,22,25-33,36,37$. These data are plotted together with ours in Fig. 4(a). The dashed line shows the correlation $U_{0}^{i n t} \propto T_{c}^{3.5}$. For different systems, the exponent $n$ may vary a little bit, for example for the $\mathrm{Ba}_{1-x} \mathrm{~K}_{x} \mathrm{Fe}_{2} \mathrm{As}_{2}, n$ is slightly smaller than that in $\mathrm{Ba}\left(\mathrm{Fe}_{1-x} \mathrm{~T}_{x}\right)_{2} \mathrm{As}_{2}$ ( $\mathrm{T}=\mathrm{Co}$ and $\mathrm{Ni}$ ). However, a global scaling law can be roughly satisfied with the exponent $n \approx 3-4$. Because the fermionic DOS should be weakly dependent on the doping level across the optimally doped point, the BCS theory implies that the condensation energy should scale roughly with $T_{c}^{2}$, which is very different from our result. We should mention that some published results from samples (mostly in the 111 system) with broad superconducting transitions are not included here. It is thus very curious to know whether more data points from variety of systems are also obeying this scaling law. Furthermore, the SH data from the $\mathrm{K}_{x} \mathrm{Fe}_{2-y} \mathrm{Se}_{2}$ and $\mathrm{KFe}_{2} \mathrm{As}_{2}$ systems are not included. This is justified by the phase separation ${ }^{38}$ in $\mathrm{K}_{x} \mathrm{Fe}_{2-y} \mathrm{Se}_{2}$. For the $\mathrm{KFe}_{2} \mathrm{As}_{2}$ system, the $T_{c}$ is too low, which may prevent determining the condensation energy precisely ${ }^{39}$.

Taking account of the BCS theory, we can deduce the condensation energy from the known values of $\gamma_{n}^{e f f}$ and the gap $\Delta_{s}$ as well. As a first approximation, assuming a spherical Fermi surface, the condensation energy is given by $U_{0}^{c a l}=1 / 2 N_{F} \Delta_{s}^{2}$ with the DOS $N_{F}=3 \gamma_{n}^{\text {eff }} /\left(2 \pi^{2} k_{B}^{2}\right)$ with $\gamma_{n}^{\text {eff }}=\gamma_{n}-\gamma_{0}$. From this argument, the condensation energy is derived as

$$
U_{0}^{c a l}=\frac{3\left(\gamma_{n}^{e f f}\right)}{4 \pi^{2} k_{B}^{2}} \Delta_{s}^{2}
$$

Starting from above equation and the values of $\gamma_{n}^{\text {eff }}$ and the gap, we calculate the condensation energy in an alternative way for our four optimally doped samples on which both the $\gamma_{n}^{e f f}$ and $\Delta_{s}$ are available, and from the published data for other samples $9,19,21,22,25-33$. Because of the multigap feature in the iron pnictide superconductors, some samples were fit by two s-wave gaps so we used the average gap $\Delta_{s}=\sqrt{\left(\left(p_{1} \Delta_{1}\right)^{2}+\left(p_{2} \Delta_{2}\right)^{2}\right.}$. For a d-wave component, the effective gap $\Delta_{s}=\frac{\sqrt{2}}{2} \Delta_{d}$ (here $\Delta_{d}$ is the maxima of the d-wave gap) is used in the formula. The calculated data of condensation energy are plotted in Fig. 4(b). The dashed line shows the power law $U_{0}^{c a l} \propto T_{c}^{3.5}$. To our surprise, not only $U_{0}^{i n t}$, but also the calculated value of the condensation energy $U_{0}^{\text {cal }}$ also obeys the correlation $U_{0} \propto T_{c}^{n}$ with $n$ of about 3-4. The result strongly indicates that the correlation between condensation energy and $\mathrm{T}_{c}$ reveals the intrinsic property in iron based superconductors. If we look back to the BNC relation, $\Delta C /\left.T_{c}\right|_{T_{c}} \propto T_{c}^{2}$, a slight difference between our result and $\mathrm{BNC}$ relation can be found by using the BCS theory. Taking $U_{0}^{B C S}=1 / 2 N_{F} \Delta_{s}^{2}$, $\Delta_{s}=1.75 k_{B} T_{c}, N_{F}=3 \gamma_{n}^{\text {eff }} /\left(2 \pi^{2} \mathrm{k}_{B}^{2}\right)$, we have $\left.\Delta C\right|_{T_{c}}$ $=1.43 \gamma_{n}^{e f f} T_{c}=6.14 U_{0}^{B C S} / T_{c}$. This would suggest from our result that $\left.\Delta C\right|_{T_{c}} \propto T_{c}^{2.5}$. This discrepancy further suggests that the simple BCS formulas, especially those based on the weak coupling approach, cannot be used in the iron based superconductors. Nevertheless, either the power law like relation found by BNC about the $\left.\Delta C\right|_{T_{c}}$ 


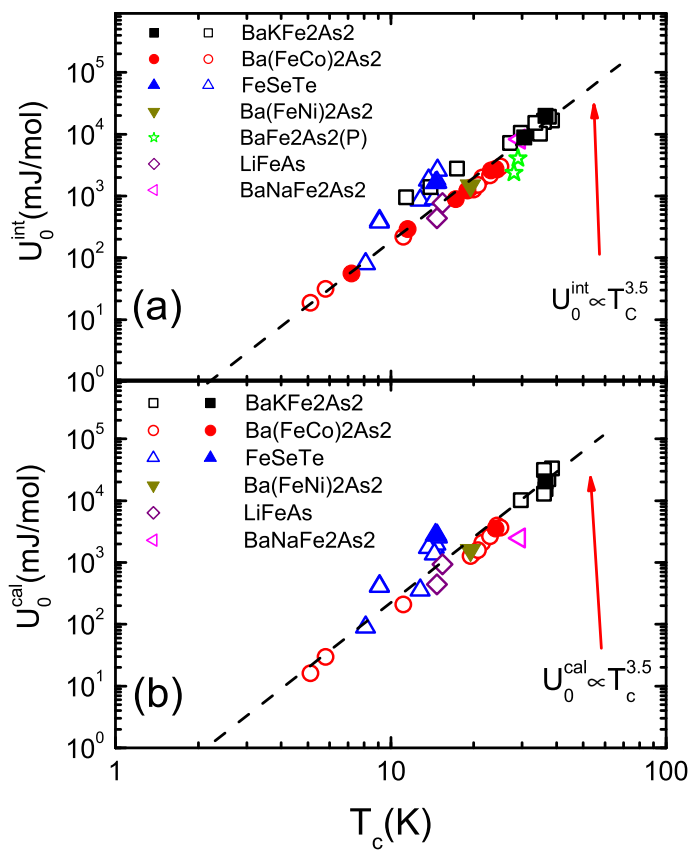

FIG. 4: (color online)Correlations between the condensation energy and $T_{c}$ in several iron based systems. Here the condensation energy is calculated through (a) integrating the entropy in the superconducting state (see text) and (b) the simple computing formula $U_{0}^{B C S}=1 / 2 N_{F} \Delta_{s}^{2}$. The dashed lines represent the relation $U_{0}^{\text {int }}$ or $U_{0}^{\text {cal }} \propto T_{c}^{3.5}$. Here the filled symbols are from our experiment, the open ones are from the available literatures.

vs. $T_{c}$, or that between the condensation energy and $T_{c}$, are beyond the expectations by the BCS theory. In the following, we argue that the doping dependence of the effective DOS (or $\gamma_{n}^{\text {eff }}$ ) may play an important role here.

Now we investigate the doping dependence of the condensation energy and the effective SH coefficient $\gamma_{n}^{\text {eff }}$ in 122 system. The results are shown in Fig. 5. It contains not only the data of our 9 samples, but also some available data from literatures. The $\mathrm{x}$-coordinate is the doped charges per Fe for every compound. In both doping sides, the quantities $U_{0}^{\text {int }} / T_{c}^{2}, U_{0}^{\text {cal }} / T_{c}^{2}$ and $\gamma_{n}^{\text {eff }}$ overlap quite well and all exhibit a maximum around the optimal doping point. Taking account the result $U_{0} \propto T_{c}^{n}$ with $n$ $=3-4$, we have $\gamma_{n}^{\text {eff }} \propto T_{c}^{m}$ with $m=1-2$. This is not expected by the BCS theory. Since $\gamma_{n}^{e f f}$ is closely related to the effective mass, we intend to argue that this novel doping dependence of $\gamma_{n}^{\text {eff }}$ (or the effective DOS) results from the mass enhancement when it is around the quantum critical point (QCP).

As we know, the antiferromagnetism and superconductivity appear closely in the electronic phase diagram revealed either by doping or by applying a high pressure in iron based superconductors. In most systems, if extrapolating the antiferromagnetic (AF) transition to

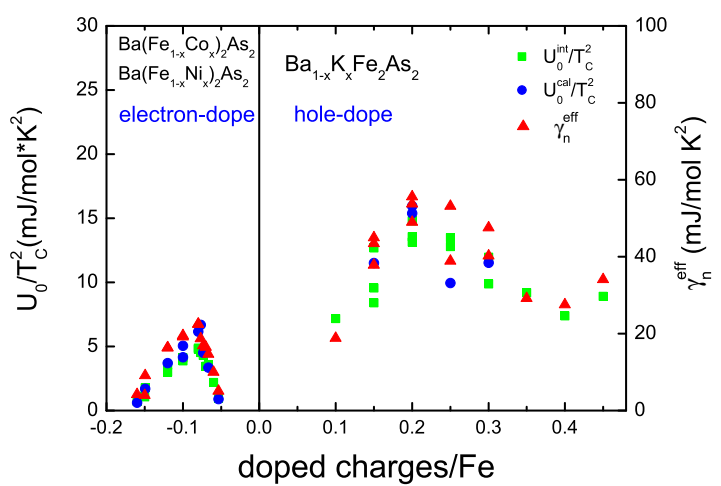

FIG. 5: (color online)Doping dependence of $U_{0}^{\text {int }} / T_{c}^{2}, U_{0}^{\text {cal }} / T_{c}^{2}$ and $\gamma_{n}^{e f f}$ for electron doped and hole doped 122 samples. The three set of data overlap each other.

zero temperature, it is found that the highest $\mathrm{T}_{c}$ appears near the point where the Neel temperature of the $\mathrm{AF}$ order becomes zero and a strong AF spin fluctuation emerges 40 . Near the optimal doping point, many novel electronic properties have been observed, for example the penetration depth seems to have a singularity in P-doped $\mathrm{BaFe}_{2} \mathrm{As}_{2}$ system ${ }^{41}$. Therefore it is quite possible that the effective mass of the electrons are strongly enhanced due to the strong coupling between the electrons and the AF spin fluctuations. This possible effect may bring about the power law like correlation between the condensation energy and $\mathrm{T}_{c}$. It was also discovered that the enhancement of effective mass appears near the quantum critical point in cuprates $\underline{42}$. The divergent effective mass was found in the heavy fermion system near the antiferromagnetic quantum critical point as well $\underline{43}$. Another feasible explanation which may be related to the above mentioned QCP mechanism is the small Fermi energy $E_{F}$ in many iron-based superconductors. In the usual situation for the BCS picture, it is known that $\omega_{D} / \mathrm{E}_{F} \ll 1$, in this case the pair-scattering occurs only near the very thin shell of the Fermi surface. While in the iron-based superconductors, there are many shallow bands crossing the Fermi level leading to a small Fermi energy $E_{F}$. This may further enhance the quantum fluctuation effect of the electronic system. Our observation here, that is $U_{0} \propto T_{c}^{n}$ with $n=3-4$, can be explained as a consequence of the QCP as argued by Zaanen ${ }^{18}$. This will certainly stimulate further theoretical and experimental efforts on this general and interesting phenomenon.

In conclusion, the SH of many iron based superconductors in the 122, 11 and 111 systems was investigated. From these data, we computed the condensation energy by two different methods and get similar power law like correlations $U_{0}^{i n t} \propto T_{c}^{n}$ and $U_{0}^{\text {cal }} \propto T_{c}^{n}$ with $n=3-4$. Combining this relationship and the semi-quantitative consideration of the BCS theory $U_{0}^{B C S}=1 / 2 N_{F} \Delta_{s}^{2}$, we find that the effective SH coefficient $\gamma_{n}^{\text {eff }}$, or the effective 
DOS is proportional to $\mathrm{T}_{c}^{m}$ with $m=1-2$ across the doping regime, either in the electron or the hole doping side. All these power law like relations are beyond the BCS understanding, but can be explained based on the QCP picture. This discovery reveals the originality that is intimately related to the unconventional superconducting mechanism.

\section{Acknowledgments}

We appreciate the useful discussions with Tao Xiang and Qimiao Si. This work is supported by the Min- istry of Science and Technology of China (973 Projects: No. 2011CBA00102, No. 2010CB923002, and No. 2012CB821403), the NSF of China, NCET project and PAPD. Work at Brookhaven was supported by DOE through contract No. DE-AC02-98CH10886.
* Electronic address: hhwen@nju.edu.cn

1 I. I. Mazin et al., Phys. Rev. Lett. 101057003 (2008).

${ }^{2}$ K. Kuroki et al., Phys. Rev. Lett. 101, 087004 (2008).

3 C. Cao et al., Phys. Rev. B 77, 220506 (2008).

${ }^{4}$ F. Wang et al., Phys. Rev. Lett. 102, 047005 (2009).

${ }^{5}$ C. Fang et al., Phys. Rev. B 77, 224509 (2008).

${ }^{6}$ Q. Si et al., Phys. Rev. Lett. 101, 076401 (2008).

7 F. Kruger et al., Phys. Rev. B 79, 054504 (2009).

${ }^{8}$ K. Haule et al., Phys. Rev. Lett. 100, 226402 (2008).

${ }^{9}$ G. Mu et al., Phys. Rev. B 79, 174501 (2009).

10 F. Hardy et al., EPL 91, 47008 (2010).

11 P. Popovich et al., Phys. Rev. Lett. 105, 027003 (2010).

12 S. L. Bud'ko et al., Phys. Rev. B 79, 220516 (2009).

13 J. S. Kim et al., Phys. Rev. B 86, 054509 (2012).

14 J. S. Kim et al., J. Phys: Condens. Matter 23, 222201 (2011).

15 S. L. Budko et al., Phys. Rev. B 89, 014510 (2014).

16 V. G. Kogan et al., Phys. Rev. B 80, 214532 (2009).

17 B. Shen et al., Phys. Rev. B 84, 184512 (2011).

18 J. Zaanen, Phys. Rev. B 80, 212502 (2009).

19 B. Zeng et al., Phys. Rev. B 85, 224515 (2012).

20 Zhijun Xu et al., Phys. Rev. B 82, 104525 (2010).

21 J. Hu et al., Phys. Rev. B 83, 134521 (2011).

22 V. Tsurkan et al., Eur. Phys. J. B 79, 289 (2011).

23 T. Klein et al., Phys. Rev. B 82, 184506 (2010).
24 A. Gunther et al., Supercond. Sci. Technol 24, 045009 (2011).

25 J.-Y. Lin et al., Phys. Rev. B 84, 220507 (2011).

26 U. Stockert et al., Phys. Rev. B 83, 224512 (2011).

27 F. Wei et al., Phys. Rev. B 81, 134527 (2010).

28 P. Popovich et al., Phys. Rev. Lett. 105, 027003 (2010).

29 F. Y. Wei et al., Phys. Rev. B 84, 064508 (2011).

${ }^{30}$ K. Gofryk et al., New J. Phys. 12023006 (2010).

31 A. K. Pramanik et al., Phys. Rev. B 84, 064525 (2011).

32 T. Noji et al., J. Phys. Soc. Jpn. 81, 054708 (2012).

33 K. Gofryk et al., Phys. Rev. B 83, 064513 (2011).

34 For a superconductor with nodal gaps, there is a residual term due to the impurity scattering effect. This should not be the case in our present samples.

35 Weidong Si et al., Nat. Commun. 4, 1347 (2013).

36 J. G. Storey et al., Phys. Rev. B 88, 144502 (2013).

37 Dong-Jin Jang et al., New J. Phys. 13023036 (2011).

38 H. H. Wen, Rep. Prog. Phys. 75, 221501 (2012).

39 J. S. Kim et al., Phys. Rev. B 83, 172502 (2011).

40 F. L. Ning et al., Phys. Rev. Lett. 104, 037001 (2010).

41 T. Shibauchi et al., arXiv:1304.6387(2013).

42 S. E. Sebastian, Proc. Natl. Acad. Sci. 107, 6175 (2010).

43 P. Coleman et al., J. Phys. Condens. Matter 13, 723 (2001). 